based mastery learning (SBML) on internal medicine residents' lumbar puncture (LP) skills, assessed neurology residents' acquired LP skills from traditional clinical education, and compared the results of SBML to traditional clinical education. Residents completed a baseline skill assessment (pretest) and following a 3-hour practice and feedback, residents completed a posttest. PGY1 internal medicine residents $(\mathrm{n}=58)$ improved from a mean of $46.3 \%$ to $95.7 \%$ after SBML ( $<<001$ ) and all met the minimum passing score (set by an expert panel) at final posttest. PGY2, 3 and 4 neurology residents $(n=36)$ from 3 medical centers completed the same simulated LP assessment without SBML. The performance of traditionally trained neurology residents was significantly lower than simulator-trained residents (mean 65.4\%, p $<0.001$ ) and only $6 \%$ met the minimum passing score. (Barsuk JH, Cohen ER, Caprio T, McGaghie WC, Simuni T, Wayne DB. Simulation-based education with mastery learning improves residents' lumbar puncture skills. Neurology 2012 Jul 10;79(2):132-7). (Response \& reprints: Dr Barsuk.E-mail: jbarsuk@nmh.org).

COMMENT. Internal medicine residents who complete SBML show improvement in LP skills, with competence superior to that of traditionally trained neurology residents. Simulation technology increases procedural skill by providing practice opportunity in a relaxed environment. The researchers recommend that residents complete simulation-based training prior to performing a clinical LP. The medical maxim that experience treating the patient translates to procedural expertise is disproven for competence in performing LP (Nathan BR, Kincaid O. Does experience doing lumbar punctures result in expertise? A medical maxim bites the dust. Neurology 2012 Jul 10;79(2):115-6). Simulator training and adherence to a procedural checklist are important in the preparation and performance of an LP by residents.

\title{
RISK OF GUILLAIN-BARRE SYNDROME AND H1N1 INFLUENZA VACCINATION
}

Researchers at Laval University, Department of Pediatric Neurology (Dr Boucher), Quebec University Hospital and other centers in Montreal, Quebec, Canada assessed the risk of Guillain-Barre syndrome (GBS) following the pandemic influenza vaccination campaign in 2009, mostly using an AS03 adjuvant vaccine. Over a 6-month period, 83 confirmed GBS cases were identified and 25 had been vaccinated against 2009 influenza A (H1N1) 8 or fewer weeks before disease onset (most (19/25) were vaccinated 4 or fewer weeks before onset). Relative risk was 1.80 for all cases during the 8 -week postvaccination period and 2.75 during the 4 -weeks postvaccination. The number of GBS cases attributable to vaccination was 2 per 1 million doses. No confirmed case of GBS was reported in the immunized age group 6mo-9years; one case occurred in the 10-19 year old immunized group (rate 1.20 per 100,000 person-years). (De Wals P, Deceuninck G, Toth E, et al. Risk of Guillain-Barre syndrome following H1N1 influenza vaccination in Quebec. JAMA 2012 Jul 11;308(2):175-81). (Respond: Philippe De Wals MD PhD, Universite Laval, Quebec. E-mail: philippe.de.wals@ssssgouv.qc.ca).

COMMENT. A small but significant risk of GBS was associated with the 2009 influenza A (H1N1) vaccination campaign in Quebec, Canada. In the US, results of the 
CDCs Emerging Infections Program showed a statistically significant association between nonadjuvant 2009 influenza A (H1N1) influenza vaccines and GBS. (Tokars JI et al. Pharmacoepidemiol Drug Saf 2012 May;21(5):546-52). The RR varied between 2.1 and 3.0; attributable risks were $1.5-2.8$ per million doses administered. In the 19761977 US epidemic, an unusually high rate of GBS followed the inactivated "swine" influenza A (H1N1) vaccination program. The variable risk of GBS with different H1N1 vaccines is an annual concern.

Increased vaccine-attributable risk for febrile convulsions following influenza vaccine. (Kelly $H$, Carcione D, Dowse GK, Effler P. Pediatr Infect Dis J 2012 Jul;31(7):792). Analysis of vaccine-attributable risk (VAR) was confined to children 6-59 months of age presenting to 9 Perth, Australia hospitals for management of a febrile convulsion in the 49 days between March 8, 2010, when the influenza vaccination program commenced and April 24, 2010, 48 hours after vaccinations for children were suspended. Ninety-nine children presented with febrile convulsions, 39 within 72 hours of receiving an influenza vaccine and 60 who had not been vaccinated. Of the 39 who had a febrile convulsion after influenza vaccination, 38 had received the 2010 vaccine, Fluvax. The total number receiving Fluvax in Perth metropolitan area was 11,963 . The 49-day risk of ED presentation for febrile convulsion following Fluvax was 38/11,963 or $32 / 10,000$. The risk in those not receiving Fluvax was $7 / 10,000$. The VAR as risk difference is $25 / 10,000$. For the corresponding period in 2009 the VAR is estimated as zero. Further use of Fluvax was suspended in Australia.

\section{MUSCLE DISORDERS}

\section{MYOTONIC DISORDERS IN AN EMG LAB OVER 12 YEARS}

Researchers at the EMG Laboratory, Boston Children's Hospital, MA assessed the spectrum of disorders associated with electrophysiologic myotonia in a pediatric electromyography laboratory. Of 2234 patients tested from 2000-2011 and screened retrospectively, 11 patients had myotonic discharges alone; 8 exhibited both myotonic discharges and myopathic motor unit potentials; and 54 demonstrated myopathic motor unit potentials alone. The diagnoses of patients with myotonic discharges alone included myotonia congenita (Thomsen disease) in 3, paramyotonia congenita in 2, congenital myopathy in 1, and Pompe disease (acid maltase deficiency) in 1; one mimicking SMA type 1 in infancy had Prader-Willi syndrome. The diagnoses of patients with both myotonic discharges and myopathic motor unit potentials included congenital myopathy in 2 and non-Pompe glycogen storage diseases in 3 . The presence of myopathic potentials helps in the differential diagnosis. (Shah DU, Darras BT, Markowitz JA, Jones HR Jr, Kang PB. The spectrum of myotonic and myopathic disorders in a pediatric electromyography laboratory over 12 years. Pediatr Neurol 2012 Aug;47(2):97-100). (Respond: Dr Kang. E-mail: peter.kang@childrens.harvard.edu).

COMMENT. Channelopathies (myotonia congenita and paramyotonia congenita), glycogen storage diseases (primarily Pompe disease), and congenital myopathies are most commonly associated with EMG myotonic discharges in childhood. 